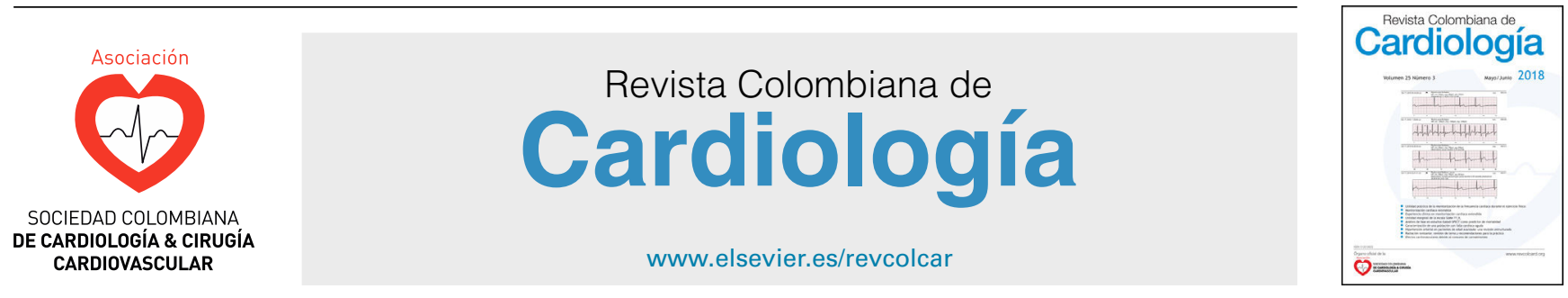

\title{
Riesgo biológico en Cardiología intervencionista
}

\author{
Diana C. Moncada-Bonilla ${ }^{a, *}$ e Isabel C. Ramírez-Sánchez ${ }^{\mathrm{b}, \mathrm{c}}$
}

\author{
a Hospital Universitario San Vicente Fundación, Medellín, Colombia \\ b Hospital Pablo Tobón Uribe, Medellín, Colombia \\ c Universidad de Antioquia, Medellín, Colombia
}

Recibido el 12 de mayo de 2019; aceptado el 15 de julio de 2019

\section{PALABRAS CLAVE \\ Exposición ocupacional; \\ Virus de la inmunodeficiencia humana; Hepacivirus; Virus de la hepatitis B; Cardiología intervencionista; Riesgo biológico}

\begin{abstract}
Resumen
Introducción: El cardiólogo intervencionista está expuesto en su práctica diaria a infecciones adquiridas por exposición ocupacional de predominante transmisión parenteral, de ahí que entender los factores de riesgo de infección, las medidas de prevención y el manejo de las exposiciones no controladas sea fundamental en la práctica clínica.

Objetivos: Revisar la magnitud del riesgo biológico al que se expone el cardiólogo intervencionista en su práctica asistencial, describir las principales medidas de prevención y reducción del riesgo y dara conocer directrices generales del manejo de la exposición ocupacional de riesgo biológico.

Métodos: Se hizo una revisión de la literatura disponible de las diferentes sociedades científicas, así como estudios clínicos publicados en las bases de datos PubMed, SciELO, MEDLINE y Google scholar. La clasificación de la evidencia se basó en los criterios del sistema GRADE (del inglés Grading of Recommendations, Assessment, Development and Evaluation).

Resultados: Se describen las recomendaciones dirigidas a la prevención de la exposición de riesgo biológico ocupacional, así como las indicaciones de manejo y profilaxis postexposición. Conclusiones: Este documento resume los principales factores de riesgo para infección de adquisición ocupacional y plantea estrategias de prevención y pautas generales para el manejo de las exposiciones no controladas.

(C) 2019 Sociedad Colombiana de Cardiología y Cirugía Cardiovascular. Publicado por Elsevier España, S.L.U. Este es un artículo Open Access bajo la licencia CC BY-NC-ND (http:// creativecommons.org/licenses/by-nc-nd/4.0/).
\end{abstract}

\section{Biological risk in Interventional cardiology}

\begin{abstract}
Introduction: The interventional cardiologists are exposed in their daily practice to infections acquired due to occupational exposure that are mainly by parenteral transmission. Thus, an understanding of the infection risk factors, prevention measures, and the management of uncontrolled exposure, should be essential in clinical practice.
\end{abstract}

\footnotetext{
* Autor para correspondencia.

Correo electrónico: diana.bonilla@sanvicentefundacion.com (D.C. Moncada-Bonilla).
} 
Hepacivirus;

Hepatitis B virus; Interventional cardiology; Biological risk
Objectives: To review the magnitude of the biological risk to which interventional cardiologists are exposed in their clinical practice, to describe the main prevention measures and reduction of the risk, as well as to provide general guidelines on the management of occupational exposure to the biological risk.

Methods: A review was performed on the literature available from the different scientific societies, as well as clinical studies published in the data bases of PubMed, SciELO, MEDLINE, and Google scholar. The classification of the evidence is based on the criteria of the GRADE system (Grading of Recommendations, Assessment, Development, and Evaluation).

Results: Recommendations directed at the prevention of occupational exposure to biological risk are presented, as well as instructions for its management and post-exposure prophylaxis. Conclusions: This document summarises the main risk factors for occupational acquired infections and established prevention strategies and general guidelines for the management of uncontrolled exposure.

(c) 2019 Sociedad Colombiana de Cardiología y Cirugía Cardiovascular. Published by Elsevier España, S.L.U. This is an open access article under the CC BY-NC-ND license (http:// creativecommons.org/licenses/by-nc-nd/4.0/).

\section{Introducción}

La fluoroscopia y los procedimientos guiados por catéter revolucionaron el manejo de la enfermedad cardiovascular al generar mayor número de procedimientos diagnósti$\cos$ y terapéuticos de naturaleza invasiva y complejidad creciente. Estos procedimientos implican la exposición potencial a sangre o fluidos corporales que puedan estar infectados con microorganismos, como hepatitis B (VHB), hepatitis $C(\mathrm{VHC})$ y virus de la inmunodeficiencia humana $(\mathrm{VIH})$. Se han descrito hasta 26 virus, 18 bacterias, 13 parásitos y 3 levaduras que han sido transmitidos por exposición ocupacional ${ }^{1}$; sin embargo, no hay recomendaciones basadas en evidencia científica para el manejo de exposiciones diferentes al VIH, VHB y VHC pues son los microorganismos de mayor transmisión y relevancia en las exposiciones ocupacionales. Cada año en Estados Unidos ocurren 400.000 exposiciones ocupacionales a patógenos de transmisión sanguínea y 1:10 trabajadores de la salud sufre una exposición de riesgo biológico por salpicaduras o punciones con aguja; estos datos representan la mitad de las exposiciones dado que el subregistro varía entre el 50 y el $67 \%$.

Este documento se construye con el objetivo de dar respuesta a las siguientes preguntas: ¿Cuál es el riesgo biológico del cardiólogo intervencionista durante los procedimientos tanto de diagnóstico como de intervención?, ¿Cuáles son las medidas de protección? y ¿Qué hacer en caso de un accidente biológico?

\section{Estrategia de búsqueda de información}

Se realizó una revisión de la literatura publicada entre 1995 y 2019, por medio de las bases de datos PubMed, MEDLINE, SciELO, Uptodate y Google Scholar; se seleccionaron los textos en inglés. Se utilizaron los siguientes términos para la búsqueda: "post-exposure prophylaxis" OR "healthcare worker" OR "occupational exposure"' OR "occupational disease" AND HIV OR HBV OR HCV OR "blood-borne pathogen" OR "interventional cardiologist"'. La evaluación de la evidencia fue clasificada con el sistema GRADE².

\section{¿Cuál es el riesgo biológico del cardiólogo intervencionista durante los procedimientos tanto de diagnóstico como de intervención?}

El principal riesgo biológico son las infecciones trasmitidas por contacto con sangre o sus derivados ${ }^{3}$. Los procedimientos percutáneos y vasculares requieren del uso de agujas y bisturíes, implementos que se asocian con una tasa de lesión por punción del 0,6\% y una frecuencia de perforación del guante del $1 \%$ para cada procedimiento. Se calcula que el promedio de punciones por año para un médico intervencionista es de 0,3 (IC 95\%: 0-1,9) ${ }^{4}$, dato que concuerda con estudios descriptivos en los cuales el 38\% de los radiólogos intervencionistas reportaron al menos una lesión con un objeto cortante durante el último año y el $90 \%$ refirió haber tenido al menos una punción en toda su carrera ${ }^{5,6}$. Todas las exposiciones accidentales implican potencial riesgo de infección; dicha exposición puede ser por punción percutánea o por exposición de piel no intacta o mucosas a sangre $\mathrm{u}$ otros fluidos visiblemente contaminados con sangre. De tal manera que una exposición ocupacional puede terminar en la adquisición de infecciones como la hepatitis $B$, hepatitis $\mathrm{C}$ y VIH especialmente. Las punciones con aguja hueca son el método más común de exposición ocupacional dando cuenta del $59 \%$ de las exposiciones, mientras que el $19 \%$ son accidentes con agujas de sutura, $19 \%$ con vidrio y el $7 \%$ heridas con bisturí. El 20\% de las exposiciones por punción ocurrieron al momento de re-encapuchar la aguja por lo tanto es una práctica completamente desaconsejada ${ }^{7}$. Otro factor que contribuye al aumento del riesgo de transmisión percutánea son las perforaciones ocultas de los guantes, que aparecen hasta en el $23 \%$ de los procedimientos que duran más de 2 horas, secundario a la manipulación de catéteres e inyectores, así como los procedimientos que incluyen 
trombólisis y angioplastia ${ }^{8}$. El riesgo de adquirir una infección viral varía en función del tipo de fluido, el mecanismo de contacto, la prevalencia de la infección en la población, el volumen inyectado, la concentración viral y la profilaxis postexposición; ejemplo de ello es que las punciones con aguja hueca confieren un riesgo superior de infección, en comparación con las agujas de sutura, pues las agujas huecas pueden albergar hasta $1 \mu \mathrm{l}$ de volumen que puede contener abundantes partículas infecciosas.

Adicionalmente, los procedimientos vasculares tienen alto riesgo de generar salpicaduras de sangre; entre el 1 al $44 \%$ de los intervencionistas manifiestan haber tenido alguna vez exposición a sangre en las mucosas y se calcula que éstas ocurren en el 6,7 al 8,7\% de los procedimientos de angiografía, especialmente durante el proceso de remoción del catéter arterial; aquellos procedimientos que duran más de 30 minutos y que requieren más de dos cambios de catéteres también se relacionan con mayor cantidad de esparcimiento de gotas. A pesar de este riesgo, solo el 32\% de los intervencionistas utilizan rutinariamente máscara facial y solo $29 \%$ utilizan gafas de protección durante los procedimientos 9 . Un estudio prospectivo de 501 procedimientos angioinvasivos demostró una frecuencia de exposición accidental del $3 \%$, de los cuales el $71 \%$ era prevenible con las medidas de protección $^{10}$.

Para el año 2015 el CDC (del inglés Centers for disease control and prevention), reportó 58 casos confirmados y 150 casos posibles de infección por $\mathrm{VIH}$ adquirido de forma ocupacional, que ocurrieron entre 1985 y 2013. En contraste, desde el año 2000 al 2012 solo ha ocurrido un caso confirmado de transmisión ocupacional de $\mathrm{VIH}^{11}$; esta disminución en el número de infecciones obedece a la reducción de la incidencia de punciones accidentales con objetos cortantes gracias al uso ampliado de las precauciones estándar, a la disposición segura de equipos cortantes y educación, así como a la disponibilidad de profilaxis farmacológica postexposición ${ }^{11}$. Pese a que los datos muestran reducción de las infecciones, la exposición a fluidos que contienen sangre puede tener consecuencias graves y de ahí la necesidad de insistir en la implementación de medidas que disminuyan el riesgo de exposición.

\section{Riesgo de transmisión del virus de inmunodeficiencia humana}

En 2016 Colombia reportó una prevalencia de infección por VIH del $0,15 \%$ en población general, la cual fue más alta en poblaciones de riesgo ${ }^{12}$. El riesgo de transmisión tras una exposición inadvertida es mayor si la fuente tiene altas concentraciones virales en sangre, no recibe tratamiento antirretroviral, el volumen de inyección es alto y la punción es profunda. Estudios prospectivos en personal de salud han demostrado que el riesgo de transmisión del VIH tras una exposición percutánea a sangre es del $0,3(0,2-0,5 \%)$, es decir 3 infecciones por cada 1.000 incidentes, y del $0,09 \%$ $(0,006-0,5 \%)$ tras exposición a mucosas ${ }^{13}$; un estudio Italiano reciente demostró una tasa de seroconversión del 0,14 al $0,43 \%{ }^{14}$. Si bien la reducción de la carga viral disminuye el riesgo de transmisión, no se elimina la posibilidad de necesitar profilaxis ni de hacer el seguimiento sugerido en exposiciones cuya fuente tiene carga viral indetectable ${ }^{15}$.

\section{Riesgo de transmisión del virus de la hepatitis $B$}

La prevalencia de infección en Estados Unidos es del 0,1 al $0,7 \%$ en población hospitalizada, y en poblaciones de riesgo puede ser tan alta como del $15 \%$. Por su parte, la tasa de transmisión del VHB depende del estado de vacunación del expuesto y los marcadores virológicos de la fuente, por lo que llega a ser del 30\% cuando la fuente tiene antígeno de superficie positivo ( $\mathrm{HBsAg}$ ) con antígeno e positivo ( $\mathrm{HBeAg}$ ) y menor al $6 \%$ cuando la fuente tiene $\mathrm{HBeAg}$ negativo; esto significa que el VHB es diez veces más transmisible que el VHC y cien veces más transmisible que el VIH, posiblemente por la capacidad del virus de sobrevivir hasta por 7 días en sangre seca o superficies con limpieza inadecuada. Se calcula que un radiólogo intervencionista sin vacunación tiene un riesgo de 1 en 100 de adquirir infección por VHB a lo largo de su carrera. Sin embargo, el número de infecciones ocupacionales ha disminuido en un 95\% después del advenimiento de la vacunación para hepatitis $B$ y la tamización en bancos de sangre, y ha pasado de 17.000 infecciones anuales en 1983 a solo 400 en $1995^{16}$; así, la vacunación de hepatitis $B$ es la intervención preventiva más importante.

\section{Riesgo de transmisión del virus de la hepatitis C}

Se calcula que la prevalencia de VHC en la población general es del 0,6 al $2 \%{ }^{14}$; es un virus con una capacidad de transmisión ineficiente al compararse con hepatitis $B$, pero de más fácil transmisión que el VIH. La tasa de infección después de una exposición percutánea es del $1,8 \%(0 \%-10 \%)^{5,17,18}$ y el 64 al $86 \%$ de los individuos infectados desarrollarán enfermedad crónica. Por su parte, el VHC puede vivir hasta dieciséis horas en las superficies. Su capacidad de transmisión está relacionada con la alta carga del ARN viral. En la tabla 1 se describen las prevalencias y la tasa de seroconversión tras la exposición ocupacional.

\section{¿Cuáles son las medidas de protección?}

Entre estas se incluyen: educación, precauciones estándar, manejo adecuado de objetos cortantes, vacunación no interrumpida de hepatitis B con demostración de títulos de anticuerpos protectores y profilaxis postexposición.

\section{Medidas de prevención}

1. Educación: entrenamiento anual al personal asistencial en prevención del riesgo, uso correcto de las precauciones estándar y gestión adecuada de residuos.

2. Precauciones estándar o universales: son un grupo de medidas que tienen como objetivo prevenir la transmisión de microorganismos tras la exposición inadvertida percutánea, mucosa o de piel no intacta a sangre o secreciones o fluido visiblemente contaminado con sangre. Estas medidas incluyen: higiene de manos, precauciones de barrera, manejo apropiado de instrumentos cortantes y medidas de higiene y desinfección. Se calcula que el $80 \%$ de las punciones con objetos cortantes son prevenibles si se utilizan las precauciones estándar y se usan de forma segura los objetos cortantes. 
Tabla 1 Prevalencia, pruebas y tratamiento de las infecciones transmitidas por sangre

\begin{tabular}{|c|c|c|c|}
\hline Patógeno & Endemicidad (\%) & $\begin{array}{l}\text { Seroconversión } \\
\text { después de la } \\
\text { punción con aguja } \\
\text { hueca }(\%)\end{array}$ & Profilaxis \\
\hline VIH & $0,2^{\mathrm{a}}$ & 0,3 & Antirretrovirales por 4 semanas \\
\hline \multirow[t]{2}{*}{$\mathrm{VHC}$} & & & Ninguna profilaxis; \\
\hline & $2-5,8^{b}$ & 1,8 & solo seguimiento \\
\hline VHB & $0,2-5,3^{b}$ & $\begin{array}{l}\text { 6-30 en ausencia } \\
\text { de inmunización }\end{array}$ & $\begin{array}{l}\text { Varía según el estado vacunación, } \\
\text { HBsAg de la fuente y niveles } \\
\text { de anti-HBS. }\end{array}$ \\
\hline
\end{tabular}

a) Lavado/higiene de manos: debe realizarse higiene de manos rutinariamente en los cinco momentos de la Organización Mundial de la Salud; además debe hacerse lavado de manos con antisépticos virucidas siempre después de cada procedimiento, al retirar los guantes y después del contacto con fluidos corporales.

b) Precauciones de barrera: se usan para evitar el contacto de la piel o mucosas con fluidos corporales, incluyen: guantes, bata de manga larga, mascarilla de protección facial y gafas; el uso de guantes disminuye un $50 \%$ del volumen inyectado en una punción. A pesar de su importancia solo el 45 al $54 \%$ de los intervencionistas interrogados utilizan de forma adecuada las precauciones de barrera ${ }^{19}$; los guantes deben ser cambiados rutinariamente después de 90 minutos del procedimiento ${ }^{20}$. Se ha demostrado que el uso de doble guante reduce en un $72 \%$ el contacto con fluidos del paciente, en $87 \%$ el contacto con sangre del paciente cuando el guante externo sufre perforaciones y hasta en un $95 \%$ la cantidad de sangre que atraviesa las dos capas de guantes, minimizándose la carga viral de una punción percutánea sin que esto altere la sensibilidad del tacto ${ }^{21-23}$.

c) Manejo apropiado de los objetos cortantes y prevención del riesgo:

- Preferir vías de administración de medicamentos diferentes a la intravenosa.

- Utilizar inyectores a chorro o sistemas intravenosos sin aguja ya que reducen el riesgo de punción con conectores entre un 62 a un $88 \%$.

- Usar dispositivos retráctiles o que se encapuchen después de su uso ya que reducen hasta un $83 \%$ los accidentes.

- Disponer los objetos cortantes en un contenedor destinado para ello cerca al área de trabajo.

\section{Precauciones del procedimiento}

- No re-encapuchar agujas en tanto sea posible. Si es necesario re-encapuchar debe hacerse con la técnica de una mano o sosteniendo la tapa con una pinza, pero no con la mano. No doblar agujas con la mano y no manipular objetos cortantes con la mano.

- Luego de usados todos los elementos afilados deben disponerse en una bandeja de procedimientos; los que sean desechables y no se vayan a reutilizar deben descartarse en recipientes apropiados teniendo cuidado de que no salga ninguna parte del dispositivo por la apertura del contenedor ${ }^{8}$.

- Los instrumentos afilados no deben entregarse directamente de un miembro del equipo al otro. Se debe esperar a que un miembro del equipo coloque el instrumento en una superficie estable y luego de que retire su mano un segundo miembro del equipo podrá levantar el instrumento.

- Las suturas deben realizarse con un portaagujas en todos los casos y debe preferirse el uso de tijeras en lugar de bisturí.

- Si un miembro del equipo sufre una lesión, el instrumento debe retirarse inmediatamente del campo y no puede ser reutilizado en el paciente.

- Se deben usar sistemas cerrados de contención de residuos.

Se deben limpiar exhaustivamente las superficies que entran en contacto con fluidos corporales (por ejemplo fluoroscopio). En la tabla 2 se resumen algunas recomendaciones de prevención.

Inmunización contra hepatitis B: todo trabajador de la salud debe recibir tres dosis de la vacuna recombinante de hepatitis $B$ (meses 0,1 y 6 ) que induce una respuesta protectora en más del 90 al $95 \%$ de los receptores saludables vacunados con tres dosis, sin requerir dosis de refuerzo y por un tiempo promedio de veinte años de duración de la inmunidad $^{24}$. Solo deben recibir dosis adicionales si no hay adecuada documentación de la vacunación. Todos los trabajadores de la salud deben tener titulación de anticuerpos uno a dos meses después de completar el esquema de vacunación. Los títulos de anti-HBS mayores a $10 \mathrm{mlU} / \mathrm{ml}$ se consideran protectores; aquellas personas que tengan títulos de anti-HBS menores a $10 \mathrm{mIU} / \mathrm{ml}$ requerirán una nueva dosis de la vacuna, con lo cual se aumentarán los títulos de anticuerpos por encima de $10 \mathrm{mUl} / \mathrm{ml}$ entre el 24-55\% de los casos, también puede repetirse el esquema de vacunación con tres dosis, con lo cual se aumentarán los títulos de anticuerpos por encima de $10 \mathrm{mUl} / \mathrm{ml}$ entre el $44-100 \%$ de los casos. Aquellas personas que después de seis dosis de la vacuna no logran títulos de anti-HBS mayores a $10 \mathrm{mIU} / \mathrm{ml}$ se denominan no respondedores y deben ser evaluados para 
Tabla 2 Recomendaciones para reducir el riesgo de lesiones en procedimientos intervencionistas

\begin{tabular}{|c|c|c|}
\hline Equipo de seguridad personal & Precauciones de salpicadura & $\begin{array}{l}\text { Manipulación segura de } \\
\text { objetos }\end{array}$ \\
\hline $\begin{array}{l}\text { Portar máscara facial, gafas y bata } \\
\text { de manga larga }\end{array}$ & $\begin{array}{l}\text { Cubrir con mantas estériles } \\
\text { el intensificador de } \\
\text { imágenes, controles } \\
\text { y pedales. }\end{array}$ & $\begin{array}{l}\text { Conocer todo el } \\
\text { instrumental y equipos }\end{array}$ \\
\hline $\begin{array}{l}\text { Usar gafas de plomo con protector } \\
\text { lateral }\end{array}$ & & $\begin{array}{l}\text { Ubicar los materiales } \\
\text { afilados en una sección } \\
\text { designada y volverles a } \\
\text { poner en el mismo sitio } \\
\text { cuando no se estén usando }\end{array}$ \\
\hline $\begin{array}{l}\text { Lavar los manos con antiséptico antes } \\
\text { y después de cada procedimiento, } \\
\text { inmediatamente después de remover } \\
\text { los guantes }\end{array}$ & $\begin{array}{l}\text { Evitar vaciar jeringas y } \\
\text { purgar los catéteres en } \\
\text { dispositivos abiertos }\end{array}$ & \\
\hline $\begin{array}{l}\text { Utilizar doble guante en cada } \\
\text { procedimiento }\end{array}$ & $\begin{array}{l}\text { Usar sistemas de inyección } \\
\text { electrónicos }\end{array}$ & Utilizar dispositivos seguros \\
\hline $\begin{array}{l}\text { Cambiar los guantes cada noventa } \\
\text { minutos. }\end{array}$ & & $\begin{array}{l}\text { No entregar directamente } \\
\text { instrumentos afilados entre } \\
\text { los miembros del equipo }\end{array}$ \\
\hline Nunca reencapuchar agujas & $\begin{array}{l}\text { Hacer limpieza de las } \\
\text { superficies con productos } \\
\text { antisépticos bactericidas }\end{array}$ & \\
\hline $\begin{array}{l}\text { Manejar las agujas con pinzas y no } \\
\text { con los dedos }\end{array}$ & & $\begin{array}{l}\text { Usar una almohadilla de } \\
\text { espuma estéril en las } \\
\text { bandejas de biopsia para } \\
\text { colocar las agujas con el } \\
\text { extremo afilado hacia abajo }\end{array}$ \\
\hline Utilizar tijeras en lugar de bisturí & $\begin{array}{l}\text { Utilizar sistemas de } \\
\text { inyección intravenosa } \\
\text { sin agujas }\end{array}$ & \\
\hline
\end{tabular}

descartar infección crónica por hepatitis $B$, en cuyo caso se medirán antígeno de superficie (HBsAg) y anticuerpos contra el core total de hepatitis $B\left(\right.$ anti-HBc) ${ }^{24}$. Si se descarta la infección se debe considerar a dicho trabajador como no respondedor a la vacunación y, por tanto, como susceptible a la hepatitis $B$.

\section{Recomendaciones}

Adherencia estricta y continua a las precauciones estándar. Recomendación fuerte, alta calidad de la evidencia.

A. Uso de equipos de protección: gafas, mascarilla, bata y guantes para prevenir la exposición. Recomendación fuerte, alta calidad de la evidencia.

$B$. Uso de guantes durante todos los procedimientos con cambio rutinario después de 90 minutos o si se sospecha perforación. Recomendación fuerte, alta calidad de la evidencia.

C. Utilización de doble guante en procedimientos vasculares. Recomendación fuerte, moderada calidad de la evidencia.

D. Manejo y disposición apropiada de los elementos cortantes. Recomendación fuerte, alta calidad de la evidencia.
E. Vacunación contra hepatitis B para todo el personal de la salud con titulación de anticuerpos uno a dos meses después de la tercera dosis de la vacuna. Recomendación fuerte, alta calidad de la evidencia.

\section{¿Qué hacer en caso de una exposición ocupacional de riesgo biológico?}

Hay lugar a una exposición ocupacional cuando el trabajador de la salud sufre una lesión cutánea con una aguja, se lacera con un objeto cortante o tiene contacto de mucosas o piel no intacta con sangre, tejidos o fluidos contaminados con sangre. La exposición ocupacional es una urgencia médica. El manejo consta de cuatro procesos que incluyen: i) Manejo inicial; ii) consejería y evaluación del riesgo; iii) pruebas de laboratorio de la fuente y el personal expuesto; iv) tratamiento apropiado y $v$ ) seguimiento.

i): Manejo inicial: lavar el sitio de la punción con agua y jabón. Dejar fluir la sangre. Los ojos y las mucosas deben ser irrigados con abundante agua o suero fisiológico. Si el accidente fue un pinchazo no debe exprimirse la zona afectada. No se recomienda el uso de antisépticos para el manejo de las heridas.

ii) Consejería y evaluación del riesgo: se debe determinar la fuente de la exposición (sangre, fluido considerado 
infeccioso, fluido con sangre o instrumental contaminado con sangre). Se debe determinar el tipo de exposición: a) si fue a piel intacta el riesgo de transmisión es nulo ${ }^{25}$; b) si ocurrió en la mucosa o en un área de piel no intacta se debe estimar el volumen del fluido de la exposición (poco o mucho y duración del contacto); c) si la exposición fue percutánea superficial o profunda; d) ¿Qué tipo de aguja estuvo involucrada en la exposición?, ¿hueca?, ¿fue utilizada para obtener sangre de una arteria o una vena? Debe reportarse la exposición no solo para tratamiento y seguimiento sino para la identificación de los riesgos en el lugar de trabajo y la evaluación de las medidas preventivas. A pesar de ello tan solo el 19 al $38 \%$ de los trabajadores de la salud reportan una exposición ocupacional ${ }^{26}$.

iii) Pruebas de laboratorio del paciente y de la fuente: se debe conocer el estado de VIH, VHB y VHC de la fuente. La prueba de VIH debe hacerse en las primeras dos horas, pero no se debe retrasar el inicio de la profilaxis. Si la fuente es positiva para $\mathrm{VIH}$ se debe considerar la carga viral, el conteo de CD4 y el tratamiento antirretroviral que recibe. Si no es posible conocer la situación serológica de la fuente, debe considerarse de alto riesgo. Debe ofrecerse profilaxis postexposición (PEP) en todo contacto percutáneo con sangre y en todo contacto con mucosas o piel no intacta con sangre; el contacto con otros líquidos que contienen sangre o potencialmente infectantes debe evaluarse en forma individual para decidir la profilaxis. En la exposición a fluidos no infectantes no se recomienda profilaxis. Se debe determinar el estatus serológico del expuesto al $\mathrm{VIH}$, VHC y VHB (anti-HBS, anti-HBC, HBsAg) y exámenes basales como hemoleucograma y función renal y hepática. Otras consideraciones: en la persona expuesta (edad, antecedentes médicos, vacunación, estado de gestación), tiempo de evolución desde la exposición, precauciones utilizadas, antecedentes de vacunación y respuesta inmunológica a la vacunación.

\section{Profilaxis postexposición a virus de la inmunodeficiencia humana}

El beneficio de la profilaxis postexposición para la prevención del VIH se demostró con el uso de la zidovudina en monoterapia, que redujo el riesgo de seroconversión en un $81 \%$ en trabajadores de la salud que habían sido expuestos vía percutánea a sangre infectada con el virus ${ }^{27}$. Debe iniciarse idealmente en las primeras 24 horas del accidente, y siempre en las primeras 72 horas. Estudios en macacos muestran que el $100 \%$ de los animales que recibieron PEP en las primeras 24 horas no se infectaron, comparado con el $50 \%$ de los que la recibieron en las primeras 48 horas y el $25 \%$ en las primeras 72 horas $^{28}$; sin embargo, aún no se ha definido el intervalo máximo a partir del cual no hay beneficio de la PEP. En exposiciones de alto riesgo se sugiere que iniciar PEP podría ser útil hasta una semana después de la exposición. La profilaxis debe ser con tres medicamentos antirretrovirales según la última recomendación del CDC y aunque se desconoce la duración óptima, datos en el modelo animal sugieren que cuatro semanas es un esquema protector. Con el uso de los antirretrovirales de generaciones anteriores se describían tasas de abandono del régimen hasta del $87,8 \%$ en relación con eventos adversos; no obstante, la tasa de abandono se ha reducido a menos del $10 \%$ con los nuevos antirretrovirales aduciendo la mejor tolerancia ${ }^{29,30}$. En caso de que se sospeche un virus resistente debe consultarse a un experto, pero no debe retrasarse el inicio de la profilaxis convencional. La profilaxis debe interrumpirse si se confirma que la fuente es negativa (por inmunoensayo de tercera o cuarta generación negativo) y no requiere estudios posteriores ni seguimiento ${ }^{30}$. La gestación no descarta el uso de PEP; sin embargo, se desaconseja el uso de efavirenz y de dolutegravir en mujeres en edad fértil que no estén utilizando un método de anticoncepción efectiva ni en mujeres en el primer trimestre de gestación por su asociación con defectos en el tubo neural. En la tabla 3 se exponen los esquemas utilizados en PEP.

\section{Profilaxis postexposición a virus de la hepatitis B}

Se debe conocer el HBsAg de la fuente; si es desconocido se debe considerar como positivo. Los trabajadores de la salud susceptibles a la infección por hepatitis B (aquellos con anti-HBS menor a $10 \mathrm{mUl} / \mathrm{ml}$ ) deben recibir profilaxis con inmunoglobulina hiperinmune de hepatitis $B$ (HBIG), una o dos dosis de $0,06 \mathrm{ml} / \mathrm{kg}$ (dependiendo del caso) separadas por 28 días, y debe iniciarse en las primeras 24 horas idealmente (no más allá de 7 días); adicionalmente, debe iniciarse un esquema de vacunación de hepatitis $B$ en los casos que aplique, tan pronto como sea posible (no más de 7 días después del evento $)^{31,32}$. La inmunoglobulina aplicada dentro de la primera semana de la exposición alcanza un 75\% de protección contra la infección por hepatitis $\mathrm{B}$ a una dosis de $0,06 \mathrm{ml} / \mathrm{kg}$ intramuscular y ofrece protección pasiva $y$ temporal ( 3 a 6 meses). Después de 6 meses de la exposición se deben evaluar nuevamente los títulos de los anticuerpos y la presencia del HBsAg. Si previamente se había demostrado en el trabajador vacunado anti-HBS mayor a $10 \mathrm{mIU} / \mathrm{ml}$ (respondedor) o la fuente es $\mathrm{HBsAg}$ negativo no requiere intervención. En la figura 1 se resumen las recomendaciones para el manejo de la hepatitis $B$.

\section{Profilaxis postexposición a virus de la hepatitis C}

A pesar del advenimiento de los antivirales directos para el manejo de la hepatitis $C$, no existe hasta la fecha documentación de la costo-efectividad de la profilaxis dirigida para prevención de infección por el $\mathrm{VHC}^{18}$. Debe realizarse medición de anticuerpos contra el VHC (anti-VHC) a la fuente; si es negativa, no requiere seguimiento. Si la fuente es positiva debe realizarse detección del ARN-VHC (detección de ácido ribonucleico de $\mathrm{VHC}$ ) en sangre de la fuente $\mathrm{y}$, al expuesto se le debe realizar medición de anti-VHC y alaninoaminotransferasa (ALT) basales.

\section{Seguimiento en virus de la inmudeficiencia humana}

Debe aconsejarse al trabajador de la salud expuesto utilizar anticoncepción de barrera, evitar donaciones de sangre o tejido, embarazo y si es posible no dar lactancia materna durante las primeras seis a doce semanas de la exposición. Se debe reforzar en los signos de alarma de las toxicidades medicamentosas, las posibles interacciones farmacológicas 
Tabla 3 Esquema de profilaxis postexposición (PEP)

\begin{tabular}{lll}
\hline Tratamiento de elección & Esquemas alternativos & $\begin{array}{l}\text { Usar únicamente por indicación } \\
\text { de experto }\end{array}$ \\
\hline Rategravir 400 mg cada 12 horas* o & Raltegravir o Darunavir + ritonavir o & Abacavir \\
dolutegravir 50 mg cada 24 horas & etravirina o rilpivirina o atazanavir + & Efavirenz \\
+ & ritonavir o lopinavir+ritonavir & Enfurvirtide \\
Tenofovir/emtricitabina 1 tableta al día & + & Fosamprenavir \\
& Tenofovir/emtricitabina & Maraviroc \\
& Zidovudina/lamivudina & Saquinavir \\
& & Estavudina \\
\hline
\end{tabular}

Debe preferirse por encima de dolutegravir en mujeres en edad fértil sin anticoncepción o en mujeres embarazadas.

Realizar las medidas iniciales del manejo de la punción, acudir a urgencias y reportar el evento

1. Muestra de sangre del trabajador de la salud para: Anti-VHC, Anti-VIH 1 y 2, Anti-HBS en pacientes vacunados y AntiHbc + Anti-HBS en sujetos no vacunados

2. Muestra de sangre del paciente fuente: Anti-VHC, si es positivo $\rightarrow$ RNA; Anti-VIH 1 y 2 , si es positivo $\rightarrow$ carga viral para VIH; HBSAg

3. Evaluación del riesgo de la transmisión: tipo de exposición, estado de infección de la fuente, etc. 4. Deje registro en la historia clinica

\begin{tabular}{|c|c|c|}
\hline $\begin{array}{l}\frac{\text { Fuente negativa para }}{\text { Anti-VHC } \rightarrow \text { No }} \\
\text { requiere intervención. } \\
\frac{\text { Fuente positiva para }}{\text { Anti-VHC }} \\
\text { Realizar anti-VHC } \\
\text { basal al expuesto y }\end{array}$ & $\begin{array}{c}\text { Fuente HbSAg negativa } \rightarrow \text { Revisar esquema de } \\
\text { vacunación } \\
\text { Fuente HbSAg positiva o desconocida } \\
\text { Trabajador de la salud documentado como } \\
\text { respondedor previamente } \rightarrow \text { No intervención } \\
\text { Documentación de no respondedor después de } 6 \\
\text { dosis } \rightarrow \text { Dos dosis de HBIG separadas por un mes }\end{array}$ & $\begin{array}{c}\text { Fuente negativa para VIH } \rightarrow \text { no } \\
\text { requiere intervención } \\
\text { Fuente positiva para VIH y expuesto } \\
\text { con anti-VIH negativo: } \\
\text { Ampliar información de la fuente: } \\
\text { ¿Qué tratamiento que recibe?, ¿Tiene } \\
\text { un virus resistente? ¿Cuál es la carga } \\
\text { viral? } \rightarrow \text { Iniciar PEP }\end{array}$ \\
\hline $\begin{array}{l}\text { mes post-exposición. } \\
\text { Puede acortarse la }\end{array}$ & $\begin{array}{l}\text { vacuna: Si Anti-HBS }<10 \mathrm{mUL} / \mathrm{ml} \text { dar HBIGxl e } \\
\text { iniciar revacunación. Si Anti-HBS }>10 \mathrm{mUI} / \mathrm{ml} \rightarrow\end{array}$ & $\begin{array}{l}\text { HLG, plaquetas, creatinina, AST, } \\
\text { ALT basal y a las } 2 \text { semanas }\end{array}$ \\
\hline $\begin{array}{l}\text { detección midiendo } \\
\text { RNA VHC entre la 4- } \\
6 \text { semana posterior a } \\
\text { la exposición. }\end{array}$ & $\begin{array}{c}\text { No intervención } \\
\text { No vacunado o vacunación incompleta } \rightarrow \text { Si Anti-HBS } \\
<10 \text { mUL/ml dar HBIGx } 1 \text { e iniciar revacunación } \\
\text { Si requirió HBIG debe repetirse HBSAg después de } 6 \\
\text { meses de la última aplicación de la HBIG }\end{array}$ & $\begin{array}{l}\text { Repetir anti-VIH a las } 6,12 \text { semanas } \\
\text { y } 4-6 \text { meses*. Si el expuesto tiene } \\
\text { infección por VHC o la fuente tenia } \\
\text { coinfección VIH-VHC, debe } \\
\text { extenderse el seguimiento con Anti- } \\
\text { VIH hasta } 12 \text { meses }\end{array}$ \\
\hline
\end{tabular}

Figura 1 Algoritmo de manejo postexposición ocupacional.

Anti-HVC: Anticuerpos frente al virus de la hepatitis C; RNA VHC: Ácido ribonucleico de VHC; HBsAg: Antígeno de superficie de hepatitis B; Anti-VIH: Anticuerpos contra el virus del VIH; Anti-HBS: anticuerpos contra el antígeno de superficie de hepatitis B; HBIG: Inmunoglobulina hiperinmune de hepatitis B; HLG: hemoleucograma; AST: aspartato aminotransferasa; ALT: alanino aminotransferasa; PEP: Profilaxis post-exposición. *Si se utiliza inmunoensayo de 4 ta generación (antígeno-anticuerpo) se puede acortar el seguimiento a 4 meses.

y la necesidad de tener una buena adherencia. Se recomienda una evaluación temprana, en las primeras 72 horas independiente de si está tomando o no PEP para conocer información adicional de la fuente o persona expuesta. El seguimiento debe incluir prueba de VIH basal, a las 6 semanas, 12 semanas y 6 meses después de la exposición, en caso de que se utilice inmunoensayo de cuarta generación (antígeno p24 y anticuerpo) los test podrían realizarse basal, a las 6 semanas y a los 4 meses después de la exposición ${ }^{15}$. Se debe ordenar un hemoleucograma completo, función renal y hepática basal y dos semanas posteriores a la exposición. Se recomienda seguimiento extendido (12 meses) para los trabajadores de la salud infectados por hepatitis $\mathrm{C}$ o si la fuente de la exposición tenía coinfección de VIH-VHC. Siempre deberá hacerse una prueba de VIH en caso de que la persona expuesta presente síntomas compatibles con un síndrome retroviral agudo independiente del intervalo de la exposición $^{15}$. 


\section{Seguimiento en hepatitis B}

Los trabajadores respondedores a la vacuna de hepatitis B no requieren seguimiento. Los pacientes susceptibles expuestos a fuente HBsAg positiva deben usar anticoncepción de barrera, así como evitar donaciones de sangre o tejido y el embarazo. La evaluación postexposición del HBsAg debe evaluarse 6 meses después de haber recibido HBIG.

\section{Seguimiento en hepatitis C}

El tiempo de incubación de la hepatitis C varía entre 2 a 24 semanas con un promedio de 6 a 7 semanas. Se recomienda repetir los anticuerpos contra hepatitis C (anti-VHC) a los 4 a 6 meses posteriores a la exposición. Si hay disponibilidad de ARN para VHC puede realizarse 4 a 6 semanas después de la exposición para acortar el tiempo del diagnóstico; si es negativo no requiere pruebas adicionales, y si el ARN es positivo debe repetirse la prueba cada 2 meses hasta los 6 meses $^{33}$ pues hasta el 30\% de los pacientes resolverá espontáneamente la infección en las primeras 12 semanas. En la figura 1 se resumen las recomendaciones de manejo y seguimiento.

\section{Recomendaciones}

El mecanismo de transmisión de VIH, VHC y VHB se comparte y por tanto, varias de las recomendaciones.

A. Tras una exposición accidental recibir valoración lo antes posible para definir inicio de PEP. Recomendación fuerte, moderada calidad de la evidencia.

$B$. Tras una punción accidental, no exprimir la zona. Recomendación fuerte, moderada calidad de la evidencia.

C. Evaluar la fuente para conocer el estado serológico frente al VIH, VHB y VHC. Para el caso del VIH este resultado debe conocerse en las primeras 2 horas del accidente. Recomendación fuerte, alta calidad de la evidencia.

D. Intentar conocer la carga viral, el tratamiento y la historia farmacológica cuando el paciente fuente del accidente tiene infección por VIH. Recomendación fuerte, alta calidad de la evidencia

E. Considerar como exposición de alto riesgo la imposibilidad de conocer el estado serológico del paciente. Recomendación fuerte, moderada calidad de la evidencia.

F. No usar PEP en las exposiciones a fluidos corporales no infectantes. Recomendación fuerte, baja calidad de la evidencia.

G. Iniciar PEP idealmente en las primeras 24 horas y siempre en las primeras 72 horas. Recomendación fuerte, moderada calidad de la evidencia.

H. Interrumpir la PEP si se confirma que la fuente es VIH negativo. Recomendación fuerte, baja calidad de la evidencia.

I. Utilizar un esquema de tres medicamentos en todos los casos. Recomendación fuerte, baja calidad de la evidencia.

J. Evitar el uso de efavirenz y dolutegravir en mujeres en edad fértil sin anticoncepción o en embarazadas en el primer trimestre. Recomendación fuerte, moderada calidad de la evidencia.

K. Administrar en todo trabajador de la salud expuesto a hepatitis B y susceptible a la infección dos dosis de gammaglobulina hiperinmune de hepatitis $B$ separadas de 28 días, que debe iniciar en los primeros 3 días después de la exposición, seguido de la primera dosis de la vacuna de $10 \mu \mathrm{g}$ dentro de los primeros 7 días después de la exposición. Recomendación fuerte, alta calidad de la evidencia.

L. Para el caso de la hepatitis $C$ no existe PEP eficaz; debe asegurarse un diagnóstico precoz de una posible infección aguda. Recomendación fuerte, baja calidad de la evidencia.

Otras exposiciones: no existen recomendaciones específicas para el cardiólogo intervencionista respecto a la prevención de otro tipo de infecciones. El cardiólogo intervencionista debe adherirse a las precauciones de contacto, aerosol y gotas de aquellos pacientes que sean intervenidos y tengan indicación de ellas.

\section{Conclusiones}

Cada día aumenta la cantidad de procedimientos intervencionistas que implican el contacto directo con secreciones y confieren riesgo de infección por microorganismos transmitidos por sangre o sus derivados. Si bien los accidentes de riesgo biológico y las infecciones subsecuentes han disminuido en los últimos años debido al conocimiento y la divulgación de las estrategias de prevención, así como al aumento de la cobertura en vacunación de hepatitis $B$ y disponibilidad de la profilaxis postexposición para $\mathrm{VIH}$, las consecuencias de una infección ocupacional son de alta gravedad y deben evitarse a toda costa. La mejor medida de prevención es el conocimiento y uso continuo de las precauciones estándar y el manejo apropiado de los objetos cortantes. Si pese a las medidas de prevención se presenta una exposición de riesgo biológico ocupacional debe reportarse el evento para tomar las medidas correctivas e iniciar el protocolo de manejo y seguimiento que tenga lugar según el riesgo de la exposición y la evaluación serológica de la fuente.

\section{Creative commons}

Creative commons reconocimiento-no comercial-sin obra derivada (CC BY-NC-ND).

\section{Conflicto de intereses}

Ninguno.

\section{Bibliografía}

1. Aguayo-Albasini JL, Flores-Pastor B, Soria-Aledo V. Sistema GRADE: clasificación de la calidad de la evidencia y graduación de la fuerza de la recomendación. Cir Esp. 2014;92:82-8.

2. Tarantola A, Abiteboul D, Rachline A. Infection risks following accidental exposure to blood or body fluids in health care wor- 
kers: A review of pathogens transmitted in published cases. Am J Infect Control. 2006;34:367-75.

3. Smilowitz NR, Balter S, Weisz G. Occupational hazards of interventional cardiology. Cardiovasc Revasc Med. 2013;14: 223-8.

4. Hansen ME, Chair S, Bakal CW, Dixon GD, Eschelman DJ, Horton KM, et al. Guidelines Regarding HIV and Other Bloodborne Pathogens in Vascular/Interventional Radiology. J Vasc Interv Radiol. 2003;14:S375-84.

5. Tso DK, Athreya S. Reducing Blood-borne Exposure in Interventional Radiology: What the IR Should Know. Cardiovasc Intervent Radiol. 2013;36:913-6.

6. Beltrami EM, Williams IT, Shapiro CN, Chamberland ME. Risk and management of blood-borne infections in health care workers. Clin Microbiol Rev. 2000;13:385-407.

7. Do AN, Ciesielski CA, Metler RP, Hammett TA, Li J, Fleming PL. Occupationally Acquired Human Immunodeficiency Virus (HIV) Infection: National Case Surveillance Data During 20 Years of the HIV Epidemic in the United States. Infect Control Hosp Epidemiol. 2003;24:86-96.

8. Vijayananthan A, Tan L, Owen A, Bhat R, Edwards R, Robertson I, et al. Accidental blood exposure: risk and prevention in interventional radiology. Biomed Imaging Interv J. 2006;2:e55.

9. Hansen ME, McIntire DD, Miller GL, Redman HC. Use of universal precautions in interventional radiology: results of a national survey. Am J Infect Control. 1994;22:1-5.

10. Hansen ME, Miller GL, Redman HC, Mclntire DD. Needlestick injuries and blood contacts during invasive radiologic procedures: frequency and risk factors. Am J Roentgenol. 1993;160:1119-22.

11. Joyce MP, Kuhar D, Brooks JT. Occupationally acquired HIV infection among health care workers-United States, 1985-2013. 2015;63:1245.

12. Hansen ME. Bloodborne pathogens and procedure safety in interventional radiology. Semin Ultrasound CT MRI. 1998;19:209-14.

13. U.S. Public Health Service. Updated U.S. Public Health Service Guidelines for the Management of Occupational Exposures to HBV, HCV, and HIV and Recommendations for Postexposure Prophylaxis. MMWR Recomm Rep Morb Mortal Wkly Rep Recomm Rep. 29 de junio de 2001; 50(RR-11):1-52. $\backslash$.

14. Ippolito G, Puro V, Petrosillo N, De Carli G. Surveillance of occupational exposure to bloodborne pathogens in health care workers: the Italian national programme. Euro Surveill Bull Eur Sur Mal Transm Eur Commun Dis Bull. 1999;4:33-6.

15. Kuhar DT, Henderson DK, Struble KA, Heneine W, Thomas V, Cheever LW, et al. Updated US Public Health Service Guidelines for the Management of Occupational Exposures to Human Immunodeficiency Virus and Recommendations for Postexposure Prophylaxis. Infect Control Hosp Epidemiol. 2013;34:875-92.

16. Mahoney FJ, Stewart K, Hu H, Coleman P, Alter MJ. Progress toward the elimination of hepatitis $\mathrm{B}$ virus transmission among health care workers in the United States. Arch Intern Med. 1997; 157:2601-5.

17. Mitsui T, Iwano K, Masuko K, Yamazaki C, Okamoto H, Tsuda $\mathrm{F}$, et al. Hepatitis $\mathrm{C}$ virus infection in medical personnel after needlestick accident. Hepatol Baltim Md. 1992;16:1109-14.

18. Naggie S, Holland DP, Sulkowski MS, Thomas DL. Hepatitis C Virus Postexposure Prophylaxis in the Healthcare Worker: Why
Direct-Acting Antivirals Don't Change a Thing. Graham CS, editor. Clin Infect Dis. 1 de enero de 2017;64(1):92-9.

19. Reddy P, Liebovitz D, Chrisman H, Nemcek AA, Noskin GA. Infection Control Practices among Interventional Radiologists: Results of an Online Survey. J Vasc Interv Radiol. agosto de. 2009;20:1070-4, e5.

20. Hansen ME, McIntire DD, Miller GL. Occult glove perforations: frequency during interventional radiologic procedures. Am J Roentgenol. julio de. 1992;159:131-5.

21. Korniewicz D, El-Masri M. Exploring the Benefits of Double Gloving During Surgery. AORN J. marzo de. 2012;95:328-36.

22. Tanner J, Parkinson H. Double gloving to reduce surgical crossinfection. En: The Cochrane Collaboration, editor. Cochrane Database of Systematic Reviews [Internet]. Chichester, UK: John Wiley \& Sons, Ltd; 2002 [citado 12 de marzo de 2019]. Disponible en: http://doi. wiley.com/10.1002/14651858.CD003087.

23. Twomey CL. Double gloving: a risk reduction strategy. Jt Comm J Qual Saf. julio de. 2003;29:369-78.

24. Kashyap B, Tiwari U, Prakash A, Hepatitis B. virus transmission and health-care workers: Prevention, management, and awareness toward the disease. Indian J Med Spec. 2019;10:6.

25. King KC, Strony R. Needlestick. En: StatPearls. Treasure Island (FL): StatPearls. Disponible en: http://www.ncbi.nlm.nih. gov/books/NBK493147/.

26. Adefolalu A. Needle stick injuries and health workers: A preventable menace. Ann Med Health Sci Res. 2014;4:159.

27. Cardo DM, Culver DH, Ciesielski CA, Srivastava PU, Marcus R, Abiteboul D, et al. A Case-Control Study of HIV Seroconversion in Health Care Workers after Percutaneous Exposure. N Engl J Med. 1997;337:1485-90.

28. Henderson DK. HIV Postexposure Prophylais in the 21st Century. Emerg Infect Dis. 2001;7:254-8.

29. Shubber, Z, Ford, N. Web annex G: safety and efficacy of antiretroviral drugs for post-exposure prophylaxis: updated recommendations on first-line and second-line antiretroviral regimens and post-exposure prophylaxis and recommendations on early infant diagnosis of HIV: interim guidelines.2015. Disponible en: http://www.who.int/iris/handle/10665/276498.

30. Abubakar S, Iliyasu G, Dayyab FM, Inuwa S, Tudun Wada RA, Sadiq NM, et al. Post-exposure prophylaxis following occupational exposure to HIV and hepatitis B: an analysis of a 12-year record in a Nigerian tertiary hospital. J Infect Prev. 2018;19:184-9.

31. Grady GF, Lee VA, Prince AM, Gitnick GL, Fawaz KA. Vyas. Hepatitis B immune globulin for accidental exposures among medical personnel: Final report of a multicenter controlled trial. J Infect. 1979;1:201.

32. Palmović D. Prevention of hepatitis B infection in health care workers after accidental exposure. J Infect. 1987;15:221-4.

33. Beekmann SE, Henderson DK. Occupational Exposures among Healthcare Workers: New Methods for Prevention and Recommended Postexposure Prophylaxis for HIV and Hepatitis B and C. Curr Treat Options Infect Dis. 2015;7:28-38.

34. Walser EM, Dixon RG, Silberzweig JE, Bartal G, Chao CP, Gross $\mathrm{K}$, et al. Occupational Exposure to Bloodborne Pathogens in IR-Risks Prevention, and Recommendations: A Joint Guideline of the Society of Interventional Radiology and Cardiovascular and Interventional Radiological Society of Europe. J Vasc Interv Radiol. 2014;25:327-31. 